\title{
A QUANTITATIVE SHARPENING OF MORIWAKI'S ARITHMETIC BOGOMOLOV INEQUALITY
}

\author{
N. NAUMANN
}

\begin{abstract}
A. Moriwaki proved the following arithmetic analogue of the Bogomolov unstability theorem. If a torsion-free hermitian coherent sheaf on an arithmetic surface has negative discriminant then it admits an arithmetically destabilising subsheaf. In the geometric situation it is known that such a subsheaf can be found subject to an additional numerical constraint and here we prove the arithmetic analogue. We then apply this result to slightly simplify a part of C. Soulé's proof of a vanishing theorem on arithmetic surfaces.
\end{abstract}

\section{Introduction and statement of result}

Let $K$ be a number field with ring of integers $\mathcal{O}_{K}$ and $X / \operatorname{Spec}\left(\mathcal{O}_{K}\right)$ an arithmetic surface, i.e. a regular, integral, purely two-dimensional scheme, proper and flat over $\operatorname{Spec}\left(\mathcal{O}_{K}\right)$ and with smooth and geometrically connected generic fibre. Attached to a hermitian coherent sheaf on $X$ are the usual characteristic classes with values in the arithmetic Chow-groups $\widehat{C H}^{i}(X)$ (cf. [GS1], 2.5), and in particular the discriminant of $\bar{E}$

$$
\Delta(\bar{E}):=(1-r) \hat{c}_{1}(\bar{E})^{2}+2 r \hat{c}_{2}(\bar{E}) \in \widehat{C H}^{2}(X)
$$

where $r:=\operatorname{rk}(E)$. The arithmetic degree map

$$
\widehat{\operatorname{deg}}: \widehat{C H}^{2}(X)_{\mathbb{R}} \longrightarrow \mathbb{R}
$$

is an isomorphism [GS2] and we will use the same symbol to to denote an element in $\widehat{C H}^{2}(X)_{\mathbb{R}}$ and its arithmetic degree in $\mathbb{R}$, see [GS2], 1.1 for the definition of arithmetic Chow-groups with real coefficients $\widehat{C H}^{*}(X)_{\mathbb{R}}$. Following [Mo2] we define the positive cone of $X$ to be

$$
\hat{C}_{++}(X):=\left\{x \in \widehat{C H}^{1}(X)_{\mathbb{R}} \mid x^{2}>0 \text { and } \operatorname{deg}_{K}(x)>0\right\} .
$$

Given a torsion-free hermitian coherent sheaf $\bar{E}$ of rank $r \geq 1$ on $X$ and a subsheaf $E^{\prime} \subseteq E$ we endow $E^{\prime}$ with the metric induced from $\bar{E}$ and consider the

Received by the editors June 29, 2005.

Key words and phrases. Bogomolov inequality, successive minima. 
difference of slopes

$$
\xi_{\bar{E}^{\prime}, \bar{E}}:=\frac{\hat{c}_{1}\left(\bar{E}^{\prime}\right)}{\operatorname{rk}\left(E^{\prime}\right)}-\frac{\hat{c}_{1}(\bar{E})}{r} \in \widehat{C H}^{1}(X)_{\mathbb{R}} .
$$

Recall that a subsheaf $E^{\prime} \subseteq E$ is saturated if the quotient $E / E^{\prime}$ is torsion-free. Our main result is the following.

Theorem 1. Let $\bar{E}$ be a torsion-free hermitian coherent sheaf of rank $r \geq 2$ on the arithmetic surface $X$, satisfying

$$
\Delta(\bar{E})<0 .
$$

Then there is a non-zero saturated subsheaf $\bar{E}^{\prime} \subseteq \bar{E}$ such that $\xi_{\bar{E}^{\prime}, \bar{E}} \in \hat{C}_{++}(X)$ and

$$
\xi_{\bar{E}^{\prime}, \bar{E}}^{2} \geq \frac{-\Delta}{r^{2}(r-1)}
$$

Remark 2. The existence of an $\bar{E}^{\prime} \subseteq \bar{E}$ with $\xi_{\bar{E}^{\prime}, \bar{E}} \in \hat{C}_{++}(X)$ is the main result of [Mo2] and means that $\bar{E}^{\prime} \subseteq \bar{E}$ is arithmetically destabilising with respect to any polarisation of $X$, c.f. loc. cit. for more details on this. The new contribution here is the inequality (1) which is the exact arithmetic analogue of a known geometric result, c.f. for example [HL], Theorem 7.3.4.

Remark 3. A special case of Theorem 1 appears in disguised form in the proof of [So], Theorem 2: Given a sufficiently positive hermitian line bundle $\bar{L}$ on the arithmetic surface $X$ and some non-torsion element $e \in \mathrm{H}^{1}\left(X, L^{-1}\right) \simeq$ $\operatorname{Ext}^{1}\left(L, \mathcal{O}_{X}\right), C$. Soulé establishes a lower bound for

$$
\|e\|^{2}:=\sup _{\sigma: K \hookrightarrow \mathbb{C}}\|\sigma(e)\|_{L^{2}}^{2}
$$

by considering the extension determined by $e$

$$
\overline{\mathcal{E}}: 0 \longrightarrow \overline{\mathcal{O}_{X}} \longrightarrow \bar{E} \longrightarrow \bar{L} \longrightarrow 0
$$

and suitably metrised as to have $\hat{c}_{1}(\bar{E})=\bar{L}$ and $2 \hat{c}_{2}(\bar{E})=\sum_{\sigma}\|\sigma(e)\|_{L^{2}}^{2}$, hence $\Delta(\bar{E})=-\bar{L}^{2}+2 \sum_{\sigma}\|\sigma(e)\|_{L^{2}}^{2}$ (where we write $\bar{L}=\hat{c}_{1}(\bar{L})$ following the notation of loc. cit.).

If $E_{\overline{\mathbb{Q}}}$ is semi-stable the arithmetic Bogomolov inequality concludes the proof. Otherwise, the main point is to show the existence of of an arithmetic divisor $\bar{D}$ satisfying

$$
\begin{aligned}
\operatorname{deg}_{K}(\bar{D}) & \leq \operatorname{deg}_{K}(\bar{L}) / 2 \text { and } \\
2(\bar{L}-\bar{D}) \bar{D} & \leq[K: \mathbb{Q}] \cdot\|e\|^{2},
\end{aligned}
$$


c.f. (28) and (32) of loc. cit. where these inequalities are established by some direct argument. We wish to point out that the existence of some $\bar{D}$ satisfying (2) and (3) is a special case of Theorem 1. In fact, let $\bar{E}^{\prime} \subseteq \bar{E}$ be as in Theorem 1 and define $\bar{D}:=\bar{L}-\hat{c}_{1}\left(\overline{E^{\prime}}\right)$. We then compute

$$
\xi_{\bar{E}^{\prime}, \bar{E}}=\frac{\bar{L}}{2}-\bar{D}
$$

and $\xi_{\bar{E}^{\prime}, \bar{E}} \in \hat{C}_{++}(X)$ implies (2). Furthermore, the inequality (1) in the present case reads

$$
\begin{gathered}
\xi_{\bar{E}^{\prime}, \bar{E}}^{2}=\frac{\bar{L}^{2}}{4}+\bar{D}^{2}-\bar{L} \bar{D} \geq \frac{-\Delta}{4}=\frac{\bar{L}^{2}}{4}-\frac{1}{2} \sum_{\sigma}\|\sigma(e)\|_{L^{2}}^{2}, \text { i.e. } \\
2(\bar{L}-\bar{D}) \bar{D} \leq \sum_{\sigma}\|\sigma(e)\|_{L^{2}}^{2},
\end{gathered}
$$

hence the trivial estimate $[K: \mathbb{Q}] \cdot\|e\|^{2} \geq \sum_{\sigma}\|\sigma(e)\|_{L^{2}}^{2}$ gives (3).

I would like to thank K. Künnemann for useful conversations about a preliminary draft of the present note.

\section{Proof of Theorem 1}

We collect some lemmas first. We call a short exact sequence

$$
\overline{\mathcal{E}}: 0 \longrightarrow \bar{E}^{\prime} \longrightarrow \bar{E} \longrightarrow \bar{E}^{\prime \prime} \longrightarrow 0
$$

of hermitian coherent sheaves on $X$ isometric if the metrics on $E^{\prime}$ and $E^{\prime \prime}$ are induced from the one on $E$. This implies that $\hat{c}_{1}(\bar{E})=\hat{c}_{1}\left(\bar{E}^{\prime}\right)+\hat{c}_{1}\left(\bar{E}^{\prime \prime}\right)$ (i.e. $\left.\tilde{c}_{1}(\overline{\mathcal{E}})=0\right)$. We also have

$$
\hat{c}_{2}(\bar{E})=\hat{c}_{2}\left(\bar{E}^{\prime} \oplus \bar{E}^{\prime \prime}\right)-a\left(\tilde{c}_{2}(\overline{\mathcal{E}})\right) \text { in } \widehat{C H}^{2}(X),
$$

where

$$
a: \tilde{A}^{1,1}\left(X_{\mathbb{R}}\right) \longrightarrow \widehat{C H}^{2}(X)
$$

is the usual map [SABK], chapter III.

Lemma 4. If

$$
\overline{\mathcal{E}}: 0 \longrightarrow \bar{E}^{\prime} \longrightarrow \bar{E} \longrightarrow \bar{E}^{\prime \prime} \longrightarrow 0
$$

is an isometric short exact sequence of hermitian coherent sheaves on $X$ with ranks $r^{\prime}, r, r^{\prime \prime} \geq 1$ and discriminants $\Delta^{\prime}, \Delta, \Delta^{\prime \prime}$, then

$$
\frac{\Delta^{\prime}}{r^{\prime}}+\frac{\Delta^{\prime \prime}}{r^{\prime \prime}}-\frac{\Delta}{r}=\frac{r r^{\prime}}{r^{\prime \prime}} \xi_{\bar{E}^{\prime}, \bar{E}}^{2}+2 a\left(\tilde{c}_{2}(\overline{\mathcal{E}})\right) \quad \text { in } \widehat{C H}^{2}(X)_{\mathbb{R}} .
$$


Proof. We omit the computation using the formulas for $\hat{c}_{i}(\bar{E})$ recalled above which shows that the left hand side of the stated equality equals

$$
\begin{gathered}
\hat{c}_{1}(\bar{E})^{2}\left(\frac{r-1}{r}+\frac{1-r^{\prime}}{r^{\prime}}\right)+\hat{c}_{1}\left(\bar{E}^{\prime \prime}\right)^{2}\left(\frac{r-1}{r}+\frac{1-r^{\prime \prime}}{r^{\prime \prime}}\right)+ \\
+\hat{c}_{1}\left(\bar{E}^{\prime}\right) \hat{c}_{1}\left(\bar{E}^{\prime \prime}\right)\left(\frac{2(r-1)}{r}-2\right)+2 a(\tilde{c}(\overline{\mathcal{E}})) .
\end{gathered}
$$

Similarly one writes $\xi_{\bar{E}^{\prime}, \bar{E}}^{2}$ as a rational linear combination of $\hat{c}_{1}(\bar{E})^{2}, \hat{c}_{1}\left(\bar{E}^{\prime \prime}\right)^{2}$ and $\hat{c}_{1}\left(\bar{E}^{\prime}\right) \hat{c}_{1}\left(\bar{E}^{\prime \prime}\right)$ and comparing the results, the stated formula drops out.

Lemma 5. For $\overline{\mathcal{E}}$ as in Lemma 4 and $\bar{G}^{\prime \prime} \subseteq \bar{E}^{\prime \prime}$ a saturated subsheaf of rank $s \geq 1$ carrying the induced metric, put

$$
\bar{G}:=\operatorname{ker}\left(E \longrightarrow E^{\prime \prime} \longrightarrow E^{\prime \prime} / G^{\prime \prime}\right) \subseteq \bar{E}
$$

with the induced metric. Then

$$
\xi_{\bar{G}, \bar{E}}=\frac{r^{\prime}\left(r^{\prime \prime}-s\right)}{\left(r^{\prime}+s\right) r^{\prime \prime}} \xi_{\bar{E}^{\prime}, \bar{E}}+\frac{s}{r^{\prime}+s} \xi_{\bar{G}^{\prime \prime}, \bar{E}^{\prime \prime}} \quad \text { in } \widehat{C H}^{1}(X)_{\mathbb{R}} .
$$

Observe that the coefficients in the last expression are non-negative rational numbers.

Proof. We have a commutative diagram with exact rows and columns

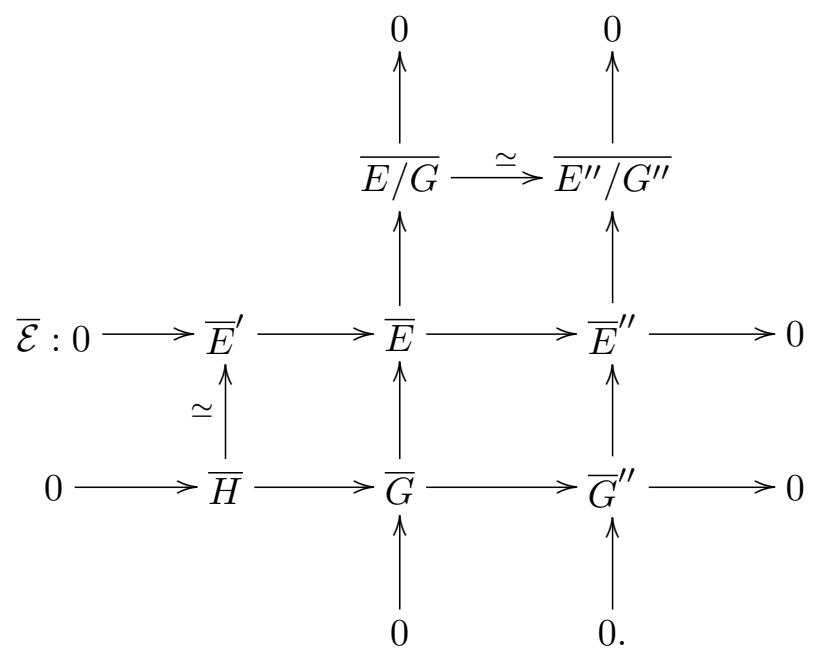

Here, we have endowed $E / G, E^{\prime \prime} / G^{\prime \prime}$ and $H$ with the metrics induced from $\bar{E}, \bar{E}^{\prime \prime}$ and $\bar{G}$, hence all rows and columns are isometric by definition. A minor point to note is that with this choice of metrics the two indicated isomorphisms are 
isometric, indeed this only means that taking sub- (resp. quotient-)metrics is transitive. One has

$$
\xi_{\bar{E}^{\prime}, \bar{E}}=\frac{r^{\prime \prime} \hat{c}_{1}\left(\bar{E}^{\prime}\right)-r^{\prime} \hat{c}_{1}\left(\bar{E}^{\prime \prime}\right)}{r^{\prime} r}
$$

and analogously for any isometric exact sequence in place of $\overline{\mathcal{E}}$. Using this and the diagram one writes both sides of the stated equality as a $\mathbb{Q}$-linear combination of $\hat{c}_{1}\left(\bar{E}^{\prime}\right), \hat{c}_{1}\left(\bar{G}^{\prime \prime}\right)$ and $\hat{c}_{1}\left(\overline{E^{\prime \prime} / G^{\prime \prime}}\right)$ to obtain the same result, namely

$$
\frac{r^{\prime \prime}-s}{\left(r^{\prime}+s\right) r} \hat{c}_{1}\left(\bar{E}^{\prime}\right)+\frac{r^{\prime \prime}-s}{\left(r^{\prime}+s\right) r} \hat{c}_{1}\left(\bar{G}^{\prime \prime}\right)-\frac{1}{r} \hat{c}_{1}\left(\overline{E^{\prime \prime} / G^{\prime \prime}}\right)
$$

Finally, we will need the following observation about the intersection theory on $X$ where, for $x \in \hat{C}_{++}(X)$, we write $|x|:=\left(x^{2}\right)^{1 / 2} \in \mathbb{R}^{+}$.

Lemma 6. The subset $\hat{C}_{++}(X) \subseteq \widehat{C H}^{1}(X)_{\mathbb{R}}$ is an open cone, i.e. $x, y \in$ $\hat{C}_{++}(X)$ and $\lambda \in \mathbb{R}^{+}$implies that $x+y, \lambda x \in \hat{C}_{++}(X)$. For $x, y \in \hat{C}_{++}$we have $|x+y| \geq|x|+|y|$.

Proof. This is [Mo2], (1.1.2.2) except for the final assertion which is obvious if $x \in \mathbb{R} y$ and we can thus assume that $V:=\mathbb{R} x+\mathbb{R} y \subseteq \widehat{C H}^{1}(X)_{\mathbb{R}}$ is twodimensional. We claim that the restriction of the intersection-pairing makes $V$ a real quadratic space of type $(1,-1)$. As we have $x \in V$ and $x^{2}>0$ we only have to exhibit some $v \in V$ with $v^{2}<0$. To achieve this let $h \in \widehat{C H}^{1}(X)_{\mathbb{R}}$ be the first arithmetic Chern class of some sufficiently positive hermitian line bundle on $X$ such that the arithmetic Hodge index theorem holds for the Lefschetz operator defined by $h$, c.f. [GS2], Theorem 2.1, ii). Then $a:=x h$ (resp. $b:=y h$ ) are non-zero real numbers for otherwise we would have $x^{2}<0$ (resp. $\left.y^{2}<0\right)$. Thus $v:=\frac{x}{a}-\frac{y}{b} \in V$ satisfies $v \neq 0$ and $v h=0$, hence $v^{2}<0$.

Fix a basis $e, f \in V$ with $e^{2}=1, f^{2}=-1$ and write

$$
\begin{gathered}
x=\alpha e+\beta f \text { and } \\
y=\gamma e+\delta f .
\end{gathered}
$$

To show that $|x+y| \geq|x|+|y|$ we can assume, changing both the signs of $x$ and $y$ if necessary, that $\alpha>0$. We then claim that $\gamma>0$. For otherwise there would be $\lambda_{1}, \lambda_{2} \in \mathbb{R}^{+}$such that $v:=\lambda_{1} x+\lambda_{2} y$ would have $e$ - coordinate equal to zero, hence $v^{2} \leq 0$ contradicting the fact that either $-v$ or $v$ lies in $\hat{C}_{++}(X)$ (depending on whether or not we changed the signs of $x$ and $y$ above).

From $x^{2}=\alpha^{2}-\beta^{2}, y^{2}=\gamma^{2}-\delta^{2}>0$ we obtain $\alpha=|\alpha| \geq|\beta|$ and $\gamma=|\gamma| \geq|\delta|$ and then $\alpha \gamma \geq|\beta \delta| \geq \beta \delta$, i.e.

$$
x y=\alpha \gamma-\beta \delta \geq 0 .
$$


To conclude, we use the following chain of equivalent statements

$$
\begin{gathered}
|x+y| \geq|x|+|y| \Leftrightarrow \\
(x+y)^{2}-(|x|+|y|)^{2} \geq 0 \Leftrightarrow \\
2 x y-2|x||y| \geq 0 \Leftrightarrow \\
x y \geq|x||y| \stackrel{(4)}{\Leftrightarrow} \\
(x y)^{2} \geq|x|^{2}|y|^{2} \Leftrightarrow \\
\alpha^{2} \gamma^{2}+\beta^{2} \delta^{2}-2 \alpha \beta \gamma \delta \geq \alpha^{2} \gamma^{2}-\alpha^{2} \delta^{2}-\beta^{2} \gamma^{2}+\beta^{2} \delta^{2} \Leftrightarrow \\
2 \alpha \beta \gamma \delta \leq \alpha^{2} \delta^{2}+\beta^{2} \gamma^{2} \Leftrightarrow \\
0 \leq(\alpha \delta-\beta \gamma)^{2} .
\end{gathered}
$$

Proof of Theorem 1. We first remark that for a torsion-free hermitian coherent sheaf $\bar{F}$ of rank one on $X$ we always have $\Delta(\bar{F}) \geq 0$. In fact,

$$
F \simeq \mathcal{L} \otimes \mathcal{I}_{Z}
$$

for some line-bundle $\mathcal{L}$ and $\mathcal{I}_{Z}$ the ideal sheaf of some closed subscheme $Z \subseteq X$ of codimension 2. This becomes an isometry for the trivial metric on $\mathcal{I}_{Z}$ and a suitable metric on $\mathcal{L}$ (since $\mathcal{I}_{Z}$ is trivial on the generic fibre of $X$ ). Then

$$
\Delta(\bar{F})=2 \hat{c}_{2}\left(\overline{\mathcal{L}} \otimes \mathcal{I}_{Z}\right)=2 \hat{c}_{2}\left(\mathcal{I}_{Z}\right)=2 \text { length }(Z) \geq 0 .
$$

By the main result of [Mo2], there is $0 \neq \bar{E}^{\prime} \subseteq \bar{E}$ saturated such that $\xi_{\bar{E}^{\prime}, \bar{E}} \in$ $\hat{C}_{++}(X)$. We can assume that, as $E^{\prime}$ varies through these subsheaves, the real numbers $\xi_{\bar{E}^{\prime}, \bar{E}}^{2}$ remain bounded for otherwise there is nothing to prove. So we can choose $0 \neq \bar{E}^{\prime} \subseteq \bar{E}$ saturated with $\xi_{\bar{E}^{\prime}, \bar{E}} \in \hat{C}_{++}(X)$ and $\xi_{\bar{E}^{\prime}, \bar{E}}^{2}$ maximal subject to these conditions. Put $E^{\prime \prime}:=E / E^{\prime}$ and consider the isometric exact sequence

$$
\overline{\mathcal{E}}: 0 \longrightarrow \bar{E}^{\prime} \longrightarrow \bar{E} \longrightarrow \bar{E}^{\prime \prime} \longrightarrow 0
$$

with discriminants $\Delta^{\prime}, \Delta, \Delta^{\prime \prime}$ and ranks $r^{\prime}, r, r^{\prime \prime}$. We claim that $\Delta^{\prime} \geq 0$. This is clear in case $r=2$ from the remark made at the beginning of the proof. In case $r \geq 3$ we assume that $\Delta^{\prime}<0$ and we let $\bar{G} \subseteq \bar{E}^{\prime}$ be a saturated subsheaf with $\xi_{\bar{G}, \bar{E}^{\prime}} \in \hat{C}_{++}$. Then $\bar{G} \subseteq \bar{E}$ is saturated and using lemma 6 we get

$$
\left|\xi_{\bar{G}, \bar{E}}\right|=\left|\xi_{\bar{G}, \bar{E}^{\prime}}+\xi_{\bar{E}^{\prime}, \bar{E}}\right| \geq\left|\xi_{\bar{G}, \bar{E}^{\prime}}\right|+\left|\xi_{\bar{E}^{\prime}, \bar{E}}\right|>\left|\xi_{\bar{E}^{\prime}, \bar{E}}\right|
$$

contradicting the maximality of $\left|\xi_{\bar{E}^{\prime}, \bar{E}}\right|$. So we have indeed $\Delta^{\prime} \geq 0$. Assume now, contrary to our assertion, that

$$
\frac{\Delta}{r}<-r(r-1) \xi_{\bar{E}^{\prime}, \bar{E}}^{2} .
$$


Then from Lemma $4, \Delta^{\prime} \geq 0,(5)$ and $\tilde{c}_{2}(\overline{\mathcal{E}}) \leq 0$ ([Mo1], 7.2) we get

$$
\begin{gathered}
\frac{\Delta^{\prime \prime}}{r^{\prime \prime}} \leq \frac{\Delta}{r}+\frac{r r^{\prime}}{r^{\prime \prime}} \xi_{\bar{E}^{\prime}, \bar{E}}^{2}<\left(-r(r-1)+\frac{r r^{\prime}}{r^{\prime \prime}}\right) \xi_{\bar{E}^{\prime}, \bar{E}}^{2} \\
=-r^{2} \frac{r^{\prime \prime}-1}{r^{\prime \prime}} \xi_{\bar{E}^{\prime}, \bar{E}}^{2} \leq 0
\end{gathered}
$$

hence $\Delta^{\prime \prime}<0$. By induction, there is $0 \neq \bar{G}^{\prime \prime} \subseteq \bar{E}^{\prime \prime}$ saturated with $\xi_{\bar{G}^{\prime \prime}, \bar{E}^{\prime \prime}} \in$ $\hat{C}_{++}(X)$ and

$$
\xi_{\bar{G}^{\prime \prime}, \bar{E}^{\prime \prime}}^{2} \geq \frac{-\Delta^{\prime \prime}}{r^{\prime \prime} 2\left(r^{\prime \prime}-1\right)}>\frac{r^{2}}{r^{\prime \prime 2}} \xi_{\bar{E}^{\prime}, \bar{E}}^{2} .
$$

Clearly $\bar{G}:=\operatorname{ker}\left(E \rightarrow E^{\prime \prime} / G^{\prime \prime}\right) \subseteq \bar{E}$ is saturated and from Lemma 5 , the positivity of the coefficients appearing there and lemma 6 we get

$$
\begin{aligned}
\left|\xi_{\bar{G}, \bar{E}}\right| & \geq \frac{r^{\prime}\left(r^{\prime \prime}-s\right)}{\left(r^{\prime}+s\right) r^{\prime \prime}}\left|\xi_{\bar{E}^{\prime}, \bar{E}}\right|+\frac{s}{r^{\prime}+s}\left|\xi_{\bar{G}^{\prime \prime}, \bar{E}^{\prime \prime}}\right| \\
& \stackrel{(6)}{>} \frac{r^{\prime}\left(r^{\prime \prime}-s\right)}{\left(r^{\prime}+s\right) r^{\prime \prime}}\left|\xi_{\bar{E}^{\prime}, \bar{E}}\right|+\frac{s}{r^{\prime}+s} \frac{r}{r^{\prime \prime}}\left|\xi_{\bar{E}^{\prime}, \bar{E}}\right| \\
& =\left(\frac{r^{\prime}\left(r^{\prime \prime}-s\right)+r s}{r^{\prime \prime}\left(r^{\prime}+s\right)}\right)\left|\xi_{\bar{E}^{\prime}, \bar{E}}\right|=\left|\xi_{\bar{E}^{\prime}, \bar{E}}\right| .
\end{aligned}
$$

This again contradicts the maximality of $\left|\xi_{\bar{E}^{\prime}, \bar{E}}\right|$ and concludes the proof.

\section{References}

[GS1] H. Gillet, C. Soulé, An arithmetic Riemann-Roch theorem, Invent. Math. 110 (1992), no. 3, 473-543.

[GS2] - Arithmetic analogs of the standard conjectures, Motives (Seattle, WA, 1991), 129-140, Proc. Sympos. Pure Math., 55, Part 1, Amer. Math. Soc., Providence, RI, 1994.

[HL] D. Huybrechts, M. Lehn, The geometry of moduli spaces of sheaves, Aspects of Mathematics, E31, Friedr. Vieweg and Sohn, Braunschweig, 1997.

[Mo1] A. Moriwaki, Inequality of Bogomolov-Gieseker type on arithmetic surfaces, Duke Math. J. 74 (1994), no. 3, 713-761.

[Mo2] B Bogomolov unstability on arithmetic surfaces, Math. Res. Lett. 1 (1994), no. 5, 601-611.

[So] C. Soulé, A vanishing theorem on arithmetic surfaces, Invent. Math. 116 (1994), no. 1-3, 577-599.

[SABK] - Lectures on Arakelov geometry, with the collaboration of D. Abramovich, J.F. Burnol and J. Kramer,Cambridge Studies in Advanced Mathematics 33, Cambridge University Press, Cambridge, 1992.

NWF I- Mathematik, Universität Regensburg, Universitätssstrasse 31,93053 REGENSBURG

E-mail address: niko.naumann@mathematik.uni-regensburg.de 